\title{
COMPOSIÇÃO E ANÁLISE ISOTÓPICA DA PRECIPITAÇÃO MENSAL EM PORTO VELHO-RO ENTRE OUTUBRO DE 2013 E MAIO DE 2014
}

\author{
J.C.Griebler Jr ${ }^{(a)}$, R.R. Ribeiro ${ }^{(b)}$, R.T. Bernardo ${ }^{(c)}$, F.E. Aquino ${ }^{(d)}$ \\ (a) Programa de Pós-Graduação em Geografia, Centro Polar e Climático, Universidade Federal do Rio Grande do Sul, \\ jose_kelso@hotmail.com \\ ${ }^{(b)}$ Departamento de Geodésia, Centro Polar e Climático, Universidade Federal do Rio Grande do Sul, \\ r.ribeiro@ufrgs.br \\ ${ }^{(c)}$ Centro Polar e Climático, Universidade Federal do Rio Grande do Sul, ronaldo.bernardo@ufrgs.br

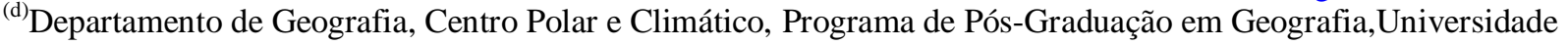 \\ Federal do Rio Grande do Sul, francisco.aquino@ufrgs.br
}

\section{Eixo:Climatologia em diferentes níveis escalares: mudanças e variabilidades}

\begin{abstract}
Resumo
O ciclo hidrológico que ocorre na Amazônia é regulado por ventos que sopram no sentido LesteOeste, responsáveis por trazer vapor d'água proveniente da evaporação do Oceano Atlântico Tropical. No interior do continente, a água precipita, evapora e recicla. Parte da água que cai sobre a Amazônia, onde está inserida Porto Velho, tem fonte no oceano e parte é proveniente de fontes internas à bacia. Este trabalho relaciona, a partir da análise de isótopos estáveis de oxigênio, os valores de $\delta 180$ medidos a partir de amostras colhidas mensalmente entre outubro de 2013 e maio de 2014, com dadosambientais de temperatura e precipitação a fim de relacionar a variação deste isótopo estável na precipitação com as variações meteorológicas locais.
\end{abstract}

Palavras chave: Ciclagem da água,Isótopos de Oxigênio, Amazônia.

\section{Introdução}

A Amazônia é uma área de grande importância para o clima mundial e, consequentemente, do continente sul-americano. A troca de energia, principalmente por convecção, que ocorre desta superfície com a atmosfera, torna-se responsável por boa parte dos regimes de precipitações locais e continentais. Desta forma, trata-se de essencial importância o conhecimento do comportamento local dos regimes de precipitação e das massas de ar que transportam umidade pela região.

Nas últimas décadas o desflorestamento da região amazônica tem aumentado consideravelmente, seja para exploração econômica (pecuária, plantações, agronegócio), seja para ocupação e uso antrópico. Tal modificação no uso e cobertura do solo são potenciais modificadores das características meteorológicas e no regime de precipitação local e circundante. Pesquisadores, como Pielke (2002), afirmam que as características da baixa atmosfera já foram afetadas e que essas mudanças no uso e cobertura do solo alteram e transformam a dinâmica e a formação de nuvens. Ray et al. (2006) observaram uma diminuição de $10 \%$ na precipitação total e de $24 \%$ na evapotranspiração real. Esses estudos expõem que o continente 
sul-americano pode e deve ser afetado, em diferentes graus, pelas modificações que ocorrem na Bacia Amazônica.

O ciclo hidrológico que ocorre na Amazônia é regulado por ventos que sopram no sentido Leste-Oeste, responsáveis por trazer vapor d'água proveniente da evaporação do Oceano Atlântico. Dentro do continente, a água precipita, evapora e recicla. Parte da água que cai sobre a Amazônia tem fonte no oceano e parte é proveniente de fontes internas à própria área.

Os isótopos de água integram informações climáticas de fonte de evaporação, local de condensação e deposição final. Quando se analisa os sinais isotópicos em amostras de precipitação de forma global, dois mecanismos dominam as variações: em altas latitudes, a temperatura controla o padrão temporal e espacial dos isótopos da água (valores do "efeito da temperatura" espacial global de $0,6-0,7 \% /{ }^{\circ} \mathrm{C}$ ) (DANSGAARD, 1964; ROZANSKIet al., 1993). Em baixas latitudes a relação entre os isótopos de água e a temperatura se desfaz e a quantidade de precipitação se torna dominante, o chamado "efeito da quantidade".

Simulações de modelo feitas por Vuilleet al. (2005) suportam a noção de que vários locais (por exemplo, sudeste da América do Sul ou o sudoeste da Bacia Amazônica próximo à encosta leste dos Andes) apresentam valores significativamente empobrecidos de $\delta^{18} \mathrm{O}$ associados a monções fortes, ainda que as precipitações sobre estas regiões sejam reduzidas. Claramente este efeito torna-se mais importante para regiões distantes, que estão localizadas mais longe das fontes de umidade. Aqui o grau de fuga durante o transporte é provavelmente mais importante do que o "efeito da quantidade" desde que forte fuga durante anos de monções ativas sobre o extremo leste da Bacia Amazônica pré-condiciona a composição de isótopos estáveis de precipitações de correntes mais baixas (VUILLEet al., 2005).

O Sistema de Monção da América do Sul (SMAS) é uma importante característica do clima sul americano e tem sido registrado em variado número de registros de isótopos estáveis pelo continente (VUILLEet al., 2005; GAN et al., 2009). Não há visão unificada de como estes registros poderiam ser interpretados. Vuilleet al. (2005) apresentaram os dados como variações interanuais na intensidade do SMAS e de ENSO influenciam $\delta^{18} \mathrm{O}$ na precipitação, baseada em ambos dados observacionais e simulações com AGCM. Há uma relação significantemente negativa entre $\delta^{18} \mathrm{O}$ e a força de SMAS sobre a Bacia Amazônica, sudeste da América do Sul e Andes Centrais. Enquanto isto é inteiramente consistente com o conhecido "efeito da quantidade" os resultados indicam que o grau de fuga para camadas superiores pode ser igualmente importante para explicar as variações interanuais em $\delta^{18} \mathrm{O}$ (VUILLEet al., 2005). 


\section{Materiais e métodos}

A coleta de amostras de água precipitada na cidade de Porto Velho, em Rondônia foi o primeiro passo realizado no trabalho da análise de dados. Esta coleta foi realizada na sede do Serviço Geológico do Brasil, a CPRM (Companhia de Pesquisa de Recursos Minerais). Para a coleta das amostras foi realizada a captação em pluviômetro próprio para esta finalidade, onde a água coletada durante a chuva fica devidamente armazenada. Para o armazenamento correto é utilizado óleo de vaselina para evitar a evaporação. Posteriormente, essas amostras, que foram recolhidas mensalmente, entre os meses de outubro de 2013 e maio de 2014, foram transferidas para um recipiente menor, de forma a minimizar o fracionamento isotópico, para ser transportadas para Porto Alegre e analisadas. De posse das amostras, foi aplicado o método de espectroscopia por tempo de decaimento em cavidade.

Para realização da medida dos isótopos de oxigênio nas amostras coletadas foi utilizado o método de espectroscopia por tempo de decaimento em cavidade, disponível no laboratório do Centro Polar e Climático (CPC), no Campus do Vale, da UFRGS. O método consiste em analisar o espectro de absorção de energia de molécula gasosa em cavidade.

A análise das amostras foi feita através da Espectroscopia por Tempo de Decaimento em Cavidade (CavityRing-Down Spectroscopy, CDRS em inglês), onde o feixe de laser (obtido a partir de um diodo de mono frequência) entra em uma cavidade com dois ou mais espelhos de alta reflexão. Os analisadores usam cavidades de três espelhos para suportar a viagem contínua da onda de luz. Isso fornece relação sinal-ruído superior quando comparada com uma cavidade de dois espelhos. Quando o laser está ligado, a cavidade é rapidamente preenchida com a luz circulante do laser. Um fotodetector sensível a pequenas quantidades de luz produz um sinal que é diretamente proporcional à intensidade de luz na cavidade, a partir de um vazamento de luz por uma abertura em um dos espelhos.

Quando o fotodetector atinge a condição de medida (em alguns décimos de microssegundos), a onda contínua do laser é abruptamente desligada. A luz que já está dentro da cavidade continua a circular entre os espelhos (aproximadamente 100.000 vezes), mas devido à refletividade dos espelhos não ser perfeita, pois atinge $99,999 \%$, a intensidade da luz na cavidade decai de forma exponencial com o tempo. Esse decaimento, chamado "ringdown", é medido em tempo real pelo fotodetector e o período de tempo para o decaimento é dado apenas em função da refletividade dos espelhos quando o valor atinge zero, quando a cavidade encontra-se sem luz. Considerando que a cavidade do espectrômetro tem apenas $25 \mathrm{~cm}$ de comprimento, o caminho efetivo da luz dentro desta pode ser superior a 20 quilômetros.

Ao se realizar a medição de isótopos de Oxigênio, o cálculo da razão isotópica (ou seja, a razão entre a 
medição e o padrão utilizado) se dá pela seguinte equação:

Onde

$$
\partial=\frac{R-R_{0}}{R_{0}}
$$

$\delta=$ medida de enriquecimento ou de empobrecimento de um isótopo em uma amostra. Como normalmente é da ordem de 0,001 se expressa seu valor em partes por mil, ou $\delta \%$, multiplicando-se seu valor por $10^{3}$.

$\mathrm{R}=$ razão isotópica da amostra

$\mathrm{R}_{0}=$ razão isotópica padrão

Em um segundo momento foi obtido os dados de análise isotópica disponibilizada pela IAEA (InternationalAtomic Energy Agency), onde o ponto de Porto Velho-RO é disponibilizado https://www.iaea.org/.Estes dados foram utilizados em comparativo com os dados obtidos a partir das análises laboratoriais das amostras obtidas em Porto Velho-RO. Os dados disponibilizados para esta cidade foram os dados do biênio 1989-1990.

Em uma terceira etapa, foi consultado o Instituto Nacional de Meteorologia (INMET), para obter dados meteorológicos de temperatura e precipitação a fim de relacionar tais dados às medidas isotópicas. De posse das medidas realizadas em laboratório e dos dados obtidos junto à IAEA, foi feita a comparação de dados para análise das semelhanças e diferenças das condições climáticas em dois períodos distintos.

Através destas relações entre os dados isotópicos e os dados meteorológicos, busca-se traçar um perfil comportamental da ocorrência da precipitação em um dado período de tempo que compreende a temporada chuvosa na Amazônia, quando o maior aporte de água abastece a maior bacia hidrográfica brasileira.

As medições dos isótopos de ${ }^{18} \mathrm{O} /{ }^{16} \mathrm{O}$ apresentam a abundância isotópica, que é registrada em notação de $\delta$ em partes por mil (\%), onde:

$$
\partial=\left(\frac{R_{A}}{R_{S}}-1\right) X 1000
$$

RA é a relação molar entre o isótopo mais raro e o isótopo mais abundante $\left(\mathrm{D} /{ }^{1} \mathrm{H}\right.$ ou $\left.{ }^{18} \mathrm{O} /{ }^{16} \mathrm{O}\right)$, e $\mathrm{RS}$ é a relação molar da amostra de interesse e um padrão internacional. O padrão internacional tanto para a análise de isótopo estável de hidrogênio quanto para isótopo estável de oxigênio é o ViennaStandard 
MeanOceanWater(VSMOW), citado anteriormente.

O Coeficiente de Pearson coeficiente é adimensional e varia de -1 a +1 . Caso os dados se alinhem perfeitamente ao longo de uma reta com declividade positiva teremos a correlação linear positiva perfeita com o coeficiente igual a 1. A correlação linear negativa perfeita ocorre quando os dados se alinham perfeitamente ao longo de uma reta com declividade negativa e o coeficiente de correlação de Pearson é igual a -1 .

Os valores intermediários apresentam a seguinte classificação:

se $0,00<\rho<0,30$, existe fraca correlação linear;

se $0,30 \leq \rho<0,60$, existe moderada correlação linear;

se $0,60 \leq \rho<0,90$, existe forte correlação linear;

se $0,90 \leq \rho<1,00$, existe correlação linear muito forte.

Dentre os fatores que afetam a intensidade do coeficiente de correlação $\rho$, bem como a precisão ao estimar a correlação $\rho$, podem ser citados o tamanho da amostra, principalmente quando é pequena, os outliers (valores discrepantes), a restrição da amplitude de uma das variáveis ou de ambas e erros de medidas. Os outliers afetam o coeficiente de correlação, principalmente quando a amostra é pequena. Quando a amostra é grande, eles têm menor efeito sobre o coeficiente de correlação. Estes podem ser detectados na análise exploratória de dados, através de teste e gráficos próprios (SACHIKO, 2004).

\section{Resultados}

A análise realizada nas amostras coletadas em Porto Velho-RO resultou nos valores da Tabela I.

Tabela I - Datas das amostragens e valores de isótopos de ${ }^{18} \mathrm{O}$ para Porto Velho-RO

\begin{tabular}{c|c|c}
\hline Data da amostragem & Mês relativo da amostra & Valor de $\delta^{18} \mathrm{O}(\%)$ \\
\hline $01 / 10 / 2013$ & Setembro/2013 & 0,48 \\
\hline $01 / 11 / 2013$ & Outubro/2013 & $-1,00$ \\
\hline $03 / 12 / 2013$ & Novembro/2013 & $-6,44$ \\
\hline $02 / 01 / 2014$ & Dezembro/2013 & $-4,93$ \\
\hline $01 / 02 / 2014$ & Janeiro/2014 & $-4,02$ \\
\hline $28 / 02 / 2014$ & Fevereiro/2014 & $-10,58$ \\
\hline $01 / 04 / 2014$ & Março/2014 & $-8,82$ \\
\hline $01 / 05 / 2014$ & Abril/2014 & $-8,04$ \\
\hline
\end{tabular}

Os resultados obtidos nestas amostragens fazem referência a características da precipitação do mês 


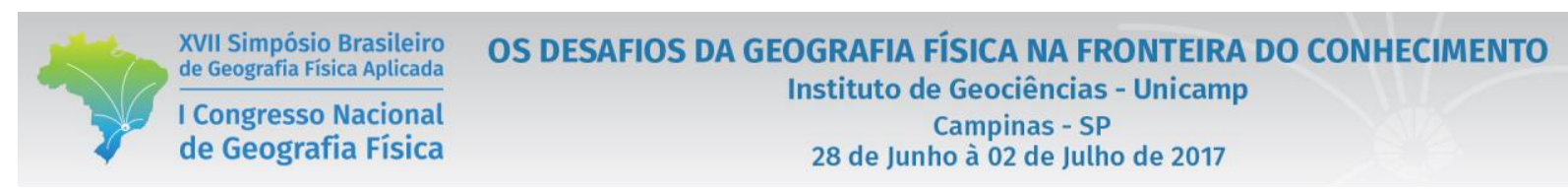

anterior à data da coleta da amostra, uma vez que o equipamento ficou recolhendo amostras pluviométricas durante todo o mês anterior ininterruptamente, até a data da coleta.

Para realizar comparativo de valores de $\delta^{18} \mathrm{O}$ medidos pela IAEA, se acessou os dados medidos pela Agência de Energia Nuclear disponíveis em seu site, acessada através do sitehttp://www.univie.ac.at/cartography/project/wiser/gui/gnip_all_index.php?sid=jose_kelso_hotmail_co m\&start_gnip=1\%27,\%27gnip_win\%27,\%29;\%22.

Osdados são referentes ao período entre os anos de 1989 e 1990. Foram coletados na estação Código OMM 8282500 (coordenadas: Lat. $-8,77^{\circ}$, Long. $-63,2^{\circ}$ e Altitude $105 \mathrm{~m}$ ) e analisados no Centro de Energia Nuclear na Agricultura da Universidade de São Paulo, em Piracicaba. De posse destes dados, comparando-se os valores medidos pela IAEA aos valores medidos na amostragem e considerando-se os respectivos meses trabalhados, chega-se aos resultados presentes na Tabela II.

Tabela II - Comparativo dos valores $\delta^{18} \mathrm{O}$ da IAEA (1989-1990) e de Porto Velho-RO (2013-2014)

\begin{tabular}{l|c|c}
\hline \multicolumn{1}{c|}{ Mês } & Valores & Valores \\
& IAEA 1989-1990 (\%) & Porto Velho-RO 2013-2014 (\%) \\
\hline Setembro & $-0,90$ & 0,48 \\
\hline Outubro & $-2,78$ & $-1,00$ \\
\hline Novembro & -590 & $-6,44$ \\
\hline Dezembro & $-3,92$ & $-4,93$ \\
\hline Janeiro & $-7,46$ & $-4,02$ \\
\hline Fevereiro & $-6,34$ & $-10,58$ \\
\hline Março & $-5,09$ & $-8,82$ \\
\hline Abril & $-6,16$ & $-8,04$ \\
\hline
\end{tabular}

Os valores apresentam certa proporcionalidade em suas grandezas, porém todos demonstram variações. Em um intervalo de 24 anos existente entre as amostragens, houve variações, não só sazonais, mas na grandeza dos valores medidos em uma mesma cidade. A relação entre os valores apresenta uma Correlação de Pearson de 0,73 , o que significa uma forte correlação entre os resultados medidos no teste com os resultados verificados em 1989-1990.

Mesmo para um período temporal que difere em 24 anos, onde muitas modificações no uso e ocupação do solo poderiam afetar significativamente no regime de chuvas da região [lembrando que cerca de $50 \%$ da pluviosidade local provém da umidade interna do sistema (MARQUESet al., 1977)], as chuvas não apresentaram significativa modificação. Marengo (2004) apontou para modificação para o regime de chuvas na Bacia Amazônica a partir do biênio 1975-76, inclusive classificando a bacia em porções Norte e Sul. Em seu trabalho, entretanto, identificou que, desde o final dos anos 1920 a Bacia Amazônica 
apresenta pequenas tendências positivas nas chuvas, mas que não são estatisticamente significativas. Os resultados isotópicos, com proporcionalidade considerável na comparação dos valores de $\delta^{18} \mathrm{O}$ em 20132014 e 1989-1990, podem apontar para este caminho, onde os conteúdos isotópicos também são semelhantes, apesar da diferença temporal significativa.

Outra análise realizada foi a relação entre os valores de $\delta^{18} \mathrm{O}$ medido nas amostras de 2013-2014 com parâmetros meteorológicos da cidade de Porto Velho-RO. Para isso foram obtidos dados meteorológicos referentes ao período entre setembro/2013 e abril/2014. Para isso foi contatado o INMET através de sua página na internet <http://www.inmet.gov.br/portal/index.php?r=home2/index >. Os dadosreferentes à estação automática Porto Velho-A925, código OMM 81932(localizada na lat. $-8,79^{\circ}$, long. $-63,85^{\circ}$, altitude $87 \mathrm{~m}$ ), em funcionamento desde 11/07/2007. Estas informações foram solicitadas ao INMET e enviadas pela SADMET (Seção de Armazenamento de Dados Meteorológicos), que as extraiu do Sistema de Informações Hidro-Meteorológica (SIM) do INMET, e enviou-as por emailsadmet.df@inmet.gov.br.Com posse desses dados, para o período da amostragem realizada, obtemos os dados da Tabela III.

Tabela III - valores meteorológicos e de $\delta^{18}$ O para período de 2013-2014

\begin{tabular}{c|c|c|c|c|c}
\hline Mês/ano & Tmédia $\left({ }^{\circ} \mathrm{C}\right)$ & Tmáx. $\left({ }^{\circ} \mathrm{C}\right)$ & Tmín. $\left({ }^{\circ} \mathrm{C}\right)$ & Precip. $(\mathrm{mm})$ & $\delta^{18} \mathrm{O}(\%)$ \\
\hline Set/2013 & 26,5 & 35,9 & 18,5 & 114,4 & 0,48 \\
\hline Out/2013 & 25,8 & 35,6 & 20,5 & 138,4 & $-1,00$ \\
\hline Nov/2013 & - & - & - & - & $-6,44$ \\
\hline Dez/2013 & 26,5 & 35,1 & 21,8 & 376,2 & $-4,93$ \\
\hline Jan/2014 & 25,6 & 34,3 & 21,6 & 411,6 & $-4,02$ \\
\hline Fev/2014 & 25,4 & 33,8 & 21,6 & 239,2 & $-10,58$ \\
\hline Mar/2014 & 25,8 & 33,6 & 21,9 & 236,2 & $-8,82$ \\
\hline Abr/2014 & 25,7 & 33,9 & 21,7 & 121,0 & $-8,04$ \\
\hline
\end{tabular}

Segundo o SADMET, possíveis falhas decorrentes de problemas (como panes) nos sensores ou no sinal de satélite são responsáveis por falhas no armazenamento ou medida dos dados. Isso ocorreu para o mês de novembro de 2013, quando nenhum dos parâmetros foi registado no banco de dados disponibilizado pelo INMET. Em virtude disso, os parâmetros meteorológicos não estão presentes na base de dados deste trabalho.

Os valores de $\delta^{18} \mathrm{O}(\%)$, relacionado com os valores de Temperatura Média, Temperatura Mínima, Temperatura Máxima e Pluviosidade Acumulada para os respectivos meses do trabalho, geraram os 
gráficos abaixo.

Os valores de $\delta^{18} \mathrm{O}$ em relação aos valores de Temperatura Média apresentam uma Correlação de Pearson de 0,60 , classificada no limiar entre a forte e a moderada correlação linear positiva. Já os valores de $\delta^{18} \mathrm{O}$ em relação às medidas de Temperatura Máxima, apresentam uma Correlação de Pearson de 0,93, classificada como muito forte correlação linear positiva. Por outro lado, os valores de $\delta^{18} \mathrm{O}$ em relação às medidas de Temperatura Mínima, apresentam uma Correlação de Pearson de -0,78, classificada forte correlação linear negativa, e os valores de $\delta^{18} \mathrm{O}$ em relação às medidas de Pluviosidade Acumulada, apresentam uma Correlação de Pearson de $-0,18$, classificada como fraca correlação linear negativa.Segundo trabalho de Marques et al. (1977) o fluxo de vapor d'água oceânico é responsável por cerca de metade da precipitação que cai sobre a Bacia Amazônica, enquanto que a outra metade é proveniente de água reciclada originada pela evapotranspiração da floresta equatorial (MOLION, 1975; VILLA NOVAet al., 1976, SALATIet al., 1979; LETTAUet al., 1979; LEOPOLDOet al., 1982). No período de verão-outono (novembro-abril) as precipitações convectivas podem dobrar ou triplicar a evapotranspiração da floresta (GROOTES E STUVIER, 1989), o que está em acordo com trabalho de Henderson et al. (1999) que, em pesquisa com testemunhos de gelo do Monte Huascarán, apontou que a relação sazonal entre $\delta^{18} \mathrm{O}$ e a temperatura, em baixas latitudes, apresenta valores mais empobrecidos de $\delta^{18} \mathrm{O}$ nos períodos mais quentes do ano, mostrando relação inversa ao comportamento ocorrido em altas latitudes. A migração para sul da ZCIT, no período quente, controla a sazonalidade Amazônica e esta é amplificada pela Baixa Pressão Boliviana, que é gerada pelo intenso aquecimento da superfície local (THOMPSONet al., 2000). Tais trabalhos apontam para um período de empobrecimento dos valores de ${ }^{18} \mathrm{O}$ na precipitação do período quente e úmido do ano na Bacia Amazônica, onde situa-se Porto VelhoRO.

A correlação dos valores de $\delta^{18} \mathrm{O}$ com as temperaturas apontam para uma relação entre essas medidas. $\mathrm{O}$ trabalho de Thompson et al. (2000) aponta para caminho semelhante, quando diz que o papel da temperatura no fracionamento de Rayleigh [modelo utilizado por Dansgaard (1964)], que assume que o condensado se forma em equilíbrio com o vapor da vizinhança, sendo assim uma simplificação do processo real, é duplo: na física molecular da mudança de fase da água, e como efeito indireto na umidade de saturação.

O condensado, mais enriquecido do que o vapor remanescente, ao precipitar, requer que o vapor circundante fique mais empobrecido de ${ }^{18} \mathrm{O}$. Este processo descreve o controle que o suprimento de vapor possui na composição isotópica da precipitação e, portanto, que os efeitos da temperatura no fracionamento na natureza não são óbvios imediatamente. 


\section{Conclusões}

O trabalho realizado com amostras colhidas em Porto Velho-RO durante 8 meses, entre 2013 e 2014, apresentou como resultado uma relação forte dos valores de $\delta^{18} \mathrm{O}$ com a temperatura local. As correlações feitas entre $\delta^{18} \mathrm{O}$, temperatura e pluviosidade apresentaram relação direta mais forte com as Temperaturas Máxima e Média, e baixa correlação com a quantidade de precipitação pluviométrica neste período analisado entre os meses de setembro de 2013 e abril de 2014. Trabalho de Thompson (2000) já indicava uma boa relação $\delta^{18} \mathrm{O}-\mathrm{T}$, quando realizou trabalho de pesquisa da fonte da umidade responsável pela recarga do manto de gelo sobre Huascarán, nos Andes Peruanos. Mesmo assim, Thompson também alerta que o efeito da temperatura no fracionamento isotópico que ocorre na natureza não é óbvio.

O efeito quantidade, que poderia ser esperado nas análises realizadas com as amostras de Porto Velho-RO, não demonstrou-se claro nesta pesquisa, haja visto a correlação entre $\delta^{18} \mathrm{O}$ e o volume de chuva ser baixo. Com isso, é possível dizer que a combinação de outros fatores (temperatura, por exemplo) fez-se mais relevante no fracionamento isotópico das amostras analisadas do que o volume de chuva.

Pode-se destacar também a relação dos valores medidos para o período set/2013-abr/2014 com os valores medidos pela IAEA no período de set/1989-abr-1990. O conjunto de valores apresentou correlação 0,73, o que significa uma forte correlação entre os resultados medidos no teste com os resultados verificados pela IAEA.

\section{Agradecimentos}

Agradecemos à geóloga Daiane Flora Hammes da CPRM, por coletar e enviar as amostras para o desenvolvimento destetrabalho, à equipe do INMET por disponibilizar os dados meteorológicos necessários, ao Técnico Químico Ronaldo TormaBernardo pelo apoio nas análises em laboratório e indicação de referências bibliográficas, ao Prof. JeffersonCardia Simões e ao Centro Polar e Climático pela infraestrutura disponibilizada para a execução deste trabalho, ao professor, Rafael Ribeiro, pela dedicação e suporte durante a execução deste trabalho, ao professor Francisco Eliseu Aquino, pelo incentivo, apoio e bibliografia indicada para a execução destetrabalho e à UFRGS pela oportunidade de usufruir de um ensino de excelência. 


\section{Bibliografia}

DANSGAARD, W. Stable isotopes in precipitation. Tellus 16, 1964, p. 436-468.

GAN, M.A., RODRIGUES, L.R. e Rao V.B. Monção na América do Sul. In: CAVALCANTI I.F.A., FERREIRA, N.J., SILVA, M.G.A.J. e DIAS, M.A.F. Tempo e clima no Brasil. São Paulo: Oficina de textos, 2009, p. 297-317.

GROOTES P.M., STUIVER M., THOMPSON L.G. e MOSLEY-THOMPSON E. Oxygenisotopechanges in tropical ice, Quelccaya, Peru.Journal of Geophysical Research n. 94, 1989, p. 1187-1194.

HENDERSON K., THOMPSON L. e LIN P-N. The recording of El Niño in ice core $\delta^{18} \mathrm{O}$ records from NevadoHuascarán, Peru. JournalofGeophysicalResearch. v. 104,1999, p. 31053-31066.

LEOPOLDO, P., FRANKEN, W. e SALATI, E. Balanço hídrico de pequena bacia hidrográfica em floresta amazônica de terra firma. ActaAmazonica, v. 12, 1982, p. 333-337.

LETTAU, H., LETTAU, K. e MOLION, L. Amazonia's hydrologic cycle and the role of atmospheric recycling in assessing deforestation effects. MonthlyWeather Review., v. 107, 1989, p 227-239.

MARENGO, J. Interdecadal variability and trends of rainfall across the Amazon Basin. Theoretical and Applied Climatology. V. 78, 2004, p. 79-96.

MARQUES, J., SANTOS, M., VILLA NOVA, N. e SALATI, E. Precipitable water and water vapor flux between Belen and Manaus, ActaAmazonica, v. 7, 1977, p. 355-362.

MOLION, L. A climatonomic study of the energy and moisture fluxes of the Amazonas basin with considerations of deforestation effects. Ph.D thesis. University of Wisconsin, Madison, 1975, 133 p.

PIELKE, R.A. Mesoscale meteorological modeling. 2nd. Edition, Academic Press, San Diego, CA. 2002. 676 p.

ROZANSKI, K., ARAGUAS-ARAGUAS L. e GONFIANTINI R.Isotopicpatterns in modern global precipitation. In: Climate Change in Continental Isotopic Records. American Geophysical Union, Geophysical Monograph 78, 1993, p. 1-36.

SALATI, E, DALL'OLIO, A., MATSUI, E. e GAT, J. Recycling of water in the Amazon Basin: an isotopic study. Water Resources Research. v. 15, 1979, p. 1250-1258.

THOMPSON, L., MOSLEY-THOMPSON E. e HENDERSON K. Ice core paleoclimate records in tropical South America since the Last Glacial Maximum. JournalofQuaternary Science. v. 15(4), 2000, p. 377-394.

VILLA NOVA, N. SALATI, E. e MATSUI, E. Estimativa da evapotranspiração na Bacia Amazônica. ActaAmazonica. v. 6, 1976, p. 215-228.

VUILLE, M. e WERNER, M. Stable isotopes in precipitation recording South American summer monsoon and ENSO variability-observations and model results. Climate Dynamics. n. 25, 2005, p. 401-413. 